\title{
Pineal hormone melatonin as an adjuvant treatment for COVID-19 (Review)
}

\author{
MARILENA VLACHOU ${ }^{1}$, ANGELIKI SIAMIDI $^{1}$, AIKATERINI DEDELOUDI $^{1}$, \\ SOFIA K. KONSTANTINIDOU ${ }^{2}$ and IOANNIS P. PAPANASTASIOU ${ }^{3}$
}

\author{
${ }^{1}$ Division of Pharmaceutical Technology, Department of Pharmacy, School of Health Sciences, \\ National and Kapodistrian University of Athens, 15784 Athens; ${ }^{2}$ First Department of Propaedeutic Internal Medicine \\ and Diabetes Center, Medical School, Laiko General Hospital, National and Kapodistrian University of Athens, \\ 11527 Athens; ${ }^{3}$ Division of Pharmaceutical Chemistry, Department of Pharmacy, School of Health Sciences, \\ National and Kapodistrian University of Athens, 15784 Athens, Greece
}

Received December 5, 2020; Accepted January 21, 2021

DOI: $10.3892 / \mathrm{ijmm} .2021 .4880$

\begin{abstract}
The beneficial properties of the pineal hormone, melatonin, as a neuroprotective and cardioprotective agent, have been previously identified. Furthermore, melatonin plays essential roles in biological rhythms resynchronization, sleep initiation/maintenance and metabolic, ocular, rheumatological diseases. In addition to these functions, melatonin is known to exert immunomodulation, anti-inflammatory and anti-oxidative effects. Due to these properties, coupled with its non-toxic nature, melatonin has been suggested to limit viral infections; however, melatonin cannot be classified as a viricidal drug. In addition, the recent increase in the number of clinical trials on melatonin's role, as an adjuvant treatment for COVID-19, has resurged the interest of the scientific community in this hormone. The present short review aimed to improve the understanding of the antiviral/anti-COVID-19 profile of melatonin and the clinical trials that have recently been conducted, with respect to its co-administration in treating individuals with COVID-19.
\end{abstract}

\section{Contents}

1. Introduction

2. Suggested mechanisms of action of melatonin against viral infections

3. Clinical trials using melatonin to treat COVID-19

4. Conclusion

Correspondence to: Dr Marilena Vlachou, Division of Pharmaceutical Technology, Department of Pharmacy, School of Health Sciences, National and Kapodistrian University of Athens, Panepistimiopolis Zografou, 15784 Athens, Greece

E-mail: vlachou@pharm.uoa.gr

Key words: SARS-CoV-2, COVID-19, melatonin, pineal hormone, mechanism of action, clinical trials

\section{Introduction}

Repurposing bioactive substances is a method, where already existing moieties, such as the pineal hormone, melatonin, are used in pharmacological studies, to determine their effect on emerging and complex diseases (1). A plethora of known drugs in circulation are being evaluated against COVID-19, as de novo drug discovery is costly and very time consuming (10 to 15 years); thus, drug repurposing significantly reduces the cost and time in treating COVID-19, in this pandemic (2). The ongoing COVID-19 pandemic continues to advance globally, as the virus spreads to new geographical areas; therefore, morbidity and mortality rates are continuously increasing (3). There is one promising drug, that has recently been identified, as a potential tool in treating patients with COVID-19, which is melatonin; thus, it is essential to examine its safety and efficacy (4).

Melatonin is biosynthesized in the pineal gland, primarily at night. It has been previously documented, that it interacts with various cellular proteins and enzymes, resulting in multiple biological effects $(5,6)$. The physiological actions of melatonin are exerted via G-protein coupled seven transmembrane cell membrane receptors (subtypes MT1 and MT2) and non-receptor-dependent mechanisms. The MT1 receptor has been associated with mammalian brain function, whilst the MT2 receptor is known to be involved in the body's circadian rhythms $(5,6)$.

Melatonin's beneficial properties, as a neuroprotective and cardioprotective agent, have been previously identified. Furthermore, melatonin plays essential roles in the female reproduction system/male fertility, resynchronization of biological rhythms, sleep initiation/maintenance and metabolism, ocular functions and rheumatological diseases (7-9). With respect to its oncostatic properties, melatonin easily interacts with the cell (both intracellularly and onto its surface receptors) to alter a broad spectrum of important pathways that are vital for its survival (10-12). These include cellular metabolism, apoptosis, circadian disruption, cell-to-cell communication and suppression of angiogenesis (12-14). In addition to these functions, melatonin is known to exert immunomodulation, 
anti-inflammatory and antioxidative effects. Due to these properties, melatonin has been hypothesized to be capable of limiting viral infections (Fig. 1) (15).

\section{Suggested mechanisms of action of melatonin against viral infections}

Melatonin cannot be classified as a viricidal drug; however, it could, indirectly, target viruses, via its anti-inflammatory, anti-oxidative and immune system enhancing actions (4). Melatonin has been used in the past, in experimental studies, both in vitro and in vivo, against viral infections with noticeable results (15-55). In particular, it has been used against the Venezuelan equine encephalitis/encephalomyelitis (VEE) virus infection, viral hepatitis, viral myocarditis, respiratory syncytial virus infection and the Ebola virus.

Patients with the VEE virus show flu-like symptoms, such as a sore throat, fever and weariness; however, in some cases, patients develop severe neurological complications, that can lead to encephalitis, chronic neurological deficits and death (16). Studies have shown that this particular virus, due to its ability to replicate in the brain, causes inflammation and subsequent blood-brain barrier disruption, leading to increased permeability, and subsequently to neuro-invasion and prolonged neurological sequelae (16-20). Furthermore, microglia respond to the resulting infection by releasing pro-inflammatory agents (21). VEE virus-infected mice brain was evaluated and the results indicated a complex immune response. Treatment with melatonin indicated that, the immunoregulatory and pro-oxidant properties of melatonin may exhibit an antiviral profile against the VEE virus by reducing oxidative stress (22). It has also been suggested that melatonin could diminish the enhanced brain expression level of apoptosis marker proteins, the concentration of malondialdehyde and nitric oxide (NO) in VEE virus-infected mice. Furthermore, the hormone may enhance the survival rate (23) and decrease $\mathrm{NO}$ and lipid peroxidation substance levels in the brain of affected animals (24). A previous study also demonstrated that melatonin could reduce NO concentrations in infected splenocytes (25). These results showed that the VEE virus-infected splenocytes generated markedly higher counts of NO in the splenic tissue. Therefore, melatonin could protect the VEE virus-infected mice by reducing NO levels $(15,25)$.

Melatonin has also been associated with acute liver failure, also known as viral hepatitis, which is characterized by jaundice, and the progression of encephalopathy, which leads to extensive liver necrosis (26). A previous study suggested that the hepatoprotective role of melatonin could be noticeably induced by activating the nuclear factor erythroid 2-related factor 2 pathway, leading to the reduction of oxidative stress and the emergence of antioxidant enzymes (27). The suppressive effects of the hormone on apoptotic damage of the liver was associated with the inhibition of endoplasmic reticulum (ER) stress, by regulating the three arms of the unfolded protein response signaling pathway $(15,26)$.

Apart from the role of melatonin in treating these viruses, the hormone has been shown to have a beneficial role in treating viral myocarditis (28). Myocarditis causes inflammation of the cardiac muscle tissues. Infectious causes of myocarditis include a wide range of parasites, such as fungi, protozoa, bacteria and viruses; however, it is most commonly associated with the inflammatory effects from viral pathogens (28). Previous studies have suggested that apart from enteroviruses and adenoviruses, such as coxsackievirus B3 (CVB3), the human herpes virus 6 and parvovirus B19, are responsible for the emergence of cardiotropic viruses $(28,29)$. The protective role of melatonin in viral myocarditis, with a focus on the Mst1-Hippo signaling pathway, ER stress and mitochondrial dysfunction, has been investigated. It was found that the hormone improved cardiac function and repressed virus-induced cardiomyocyte apoptosis. In addition, Mst1 upregulation, caused by the virus infection, was rescued by the action of melatonin (30). A previous study investigated the protective role of melatonin on viral myocarditis in vivo. Treatment of the hormone significantly healed myocardial injuries, by repressing inflammation (31). Other possible mechanisms of melatonin include the regulation of the rate of autophagy and apoptosis inhibition in mice with CVB3-induced myocarditis $(15,31,32)$.

Furthermore, melatonin has been hypothesized to affect thrombin formation and platelet physiology, caused by the Ebola virus, which increases blood coagulation and damages blood vessels, leading to hemorrhagic shock and possibly death (33). In addition, it has been suggested that melatonin increased the protein expression level of the enzyme heme oxygenase 1, which reduced the replication of the Ebola virus $(15,34,35)$.

The therapeutic effects of melatonin, in the respiratory system, have been identified, and it was found to block nuclear factor- $\mathrm{\kappa B}(\mathrm{NF}-\mathrm{\kappa B})$, the overexpression of c-Fos, and the downregulation of matrix metalloproteinases-3 (MMP-3), which leads to pro-fibrotic and pro-inflammatory cytokine production $(36,37)$. In addition, the beneficial role of melatonin in pulmonary hypertension has been associated with its antioxidant, anti-fibrotic and vasodilator effects (34).

Melatonin has also shown a protective effect on pulmonary tissues, which have been injured following acute lung injury (38). The novel analogue of melatonin, 5-hydroxy-2'-isobutyl-streptochlorin (HIS), has notably anti-inflammatory properties and has been found to inhibit the entry of immune cells into the lung, and decrease the release of pro-inflammatory cytokines, such as TNF- $\alpha$ and IL-6. These anti-inflammatory actions of HIS are mediated by the regulation of interferon- $\beta$ and toll-like receptors-dependent signaling pathways (38). Furthermore, HIS prevented the secretion of IL-1 $\beta$, by inhibiting the activation of the NLRP3 inflammasome $(38,39)$. The lipid peroxidation of the lung surfactant occurs from the production of oxygen free radicals from the activated phagocytes, which are responsible for causing acute lung injury. Melatonin, on its own or in combination with other antioxidants, reduced lipid peroxidation of the pulmonary surfactant (40). The administration of melatonin led to a notable reduction in lung lesions and the accumulation of neutrophils (a type of granulocyte, that and constitutes 40-70\% of all white blood cells in humans) and macrophages into the lungs during acute pulmonary injury. In addition, melatonin was found to inhibit the development of the NLRP3 inflammasome by suppressing extracellular histone release (41). Melatonin could act by prohibiting pulmonary fibrosis in a rat animal model, due to minimizing chloride channel activity, 


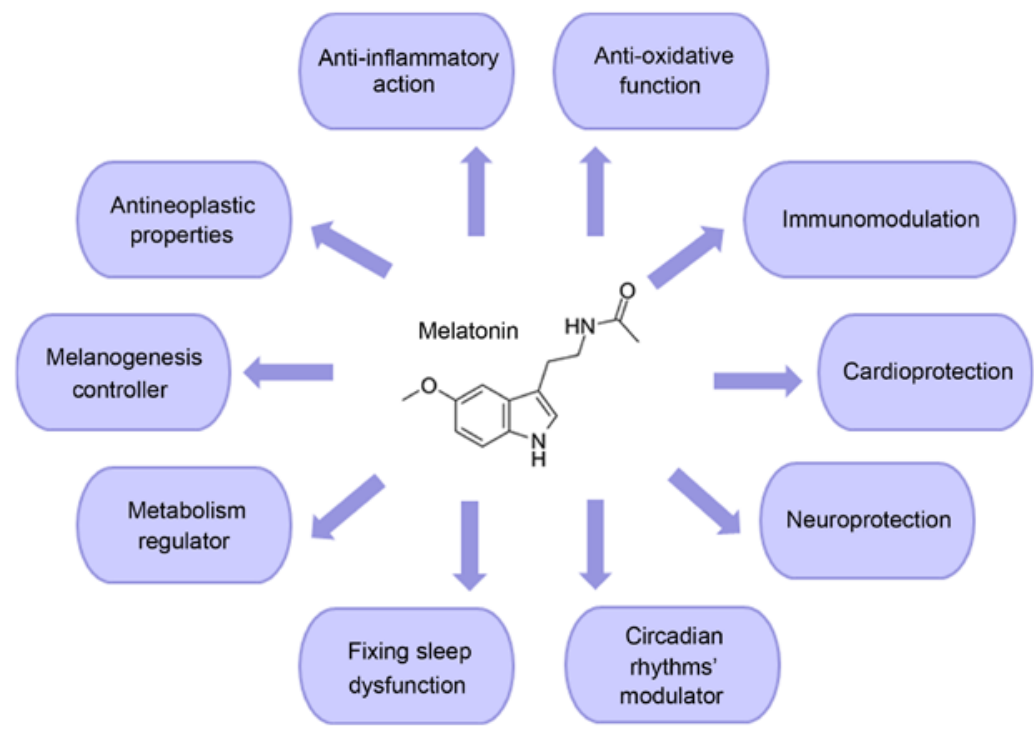

Figure 1. Schematic overview of the main functions of melatonin.

which was mediated by protein kinase C (42). In another study, melatonin modulated pneumonitis and lung fibrosis, caused by exposure to radiation by decreasing inflammatory cell infiltration, collagen deposition and alveolar thickening in the lungs (43). It is noteworthy, that melatonin and its metabolites could regulate the appropriate pro-fibrogenic and pro-inflammatory signaling pathways, which are involved in the pathophysiology of pulmonary fibrosis, leading to its protective effects on the lungs (44). Melatonin has been hypothesized to remove free radicals, and therefore may modulate autophagy pathways and apoptosis, which are critical factors in the development of idiopathic pulmonary fibrosis (10). In addition, the use of melatonin in animals (rats and rabbits), with induced hepatopulmonary syndrome, has been proven to be beneficial in the reduction of lung fibrosis levels, oxidative stress and vasodilation (45).

Pulmonary lesions, which have been associated with inflammation and oxidative stress, and are caused by nitrogen mustard in rodents, were found to be ameliorated by melatonin, as it acts as an effective reactive nitrogen and oxygen species scavenger, and as an anti-inflammatory compound (46-48). Pretreatment with melatonin, alone or in combination with quercetin in rats, with hypoxia-induced by sodium nitrite, markedly reduced the plasma levels of IL-6, tumor necrosis factor- $\alpha, \mathrm{CRP}$, heat shock protein 70 extracellular (Hsp70e), and VEGF. In addition, melatonin improved the histopathological changes in the lungs, such as improvement of the alveoli and alveolar septae, and a marked decrease in cellular infiltration, acting as a protector at the pulmonary level (49).

Melatonin has also been found to affect the protein expression level of hypoxia-inducible factor- $1 \alpha$ and NF- $\kappa \mathrm{B}$ in a murine model of hypoxic pulmonary hypertension (50). Furthermore, in vitro experiments suggested that the hormone could diminish the proliferation of pulmonary artery smooth muscle cells and the levels of phosphorylated Akt and extracellular signal-regulated kinases $1 / 2$ (50). The possible mechanistic pathways, where melatonin could affect the route of COVID-19 infection, are illustrated in Fig. 2. In view of this, the well-known melatonin's anti-inflammatory and anti-oxidative properties can aid in reducing the production of pro-inflammatory cytokines. A number of these actions are mediated by its MT1 and MT2 receptors (37,51).

In a recent study, the importance of the CD147 glycoprotein and the protective role of melatonin, which is mediated by CD147 in COVID-19, was investigated. It is known that CD147 is involved in the increase in the number of cytokines in the lungs caused by viral invasion. It was found that reduced levels of melatonin, which are found in patients who are middle-aged (40-60 years old) and elderly (>60 years old) and patients who are immune-suppressed, should be replenished by the administration of exogenous melatonin, as an adjuvant, in the treatment of COVID-19 (52).

Furthermore, the administration of the melatonin receptor drug, Ramelteon, in rats with lung injury, had various effects (53). In particular, it markedly reduced pulmonary edema, the serum concentration of 1,3-propanedial, the population of pro-inflammatory cytokines in the bronchoalveolar lavage fluid, the activation of $\mathrm{NF}-\kappa \mathrm{B}$, the protein expression level of inducible NO synthase in lung cells, and lung cells apoptosis. In addition, this drug treatment notably increased the protein expression level of intracellular protective heat shock protein 70, in lung cells, and the concentration of the anti-inflammatory cytokine, IL-10, in the bronchoalveolar lavage fluid. The results from the study recommended that the protective effects of melatonin on lung disease, were primarily mediated by its receptor (53).

In a meticulous study investigating the structure and physicochemical characteristics of melatonin, using electronic structure techniques and molecular-mechanics software, a vast amount of information was found regarding the steroelectronic characteristics of melatonin, which enabled the prediction of its bioactivity (54). In addition, the molecular docking investigation predicted the essential features of the molecules to treat the COVID-19 proteins. The maximum docking scores of $-7.28,-7.20$, and $-7.06 \mathrm{kcal} / \mathrm{mol}$ indicated that melatonin could be beneficial to protect against the viral load in vulnerable populations (54). 


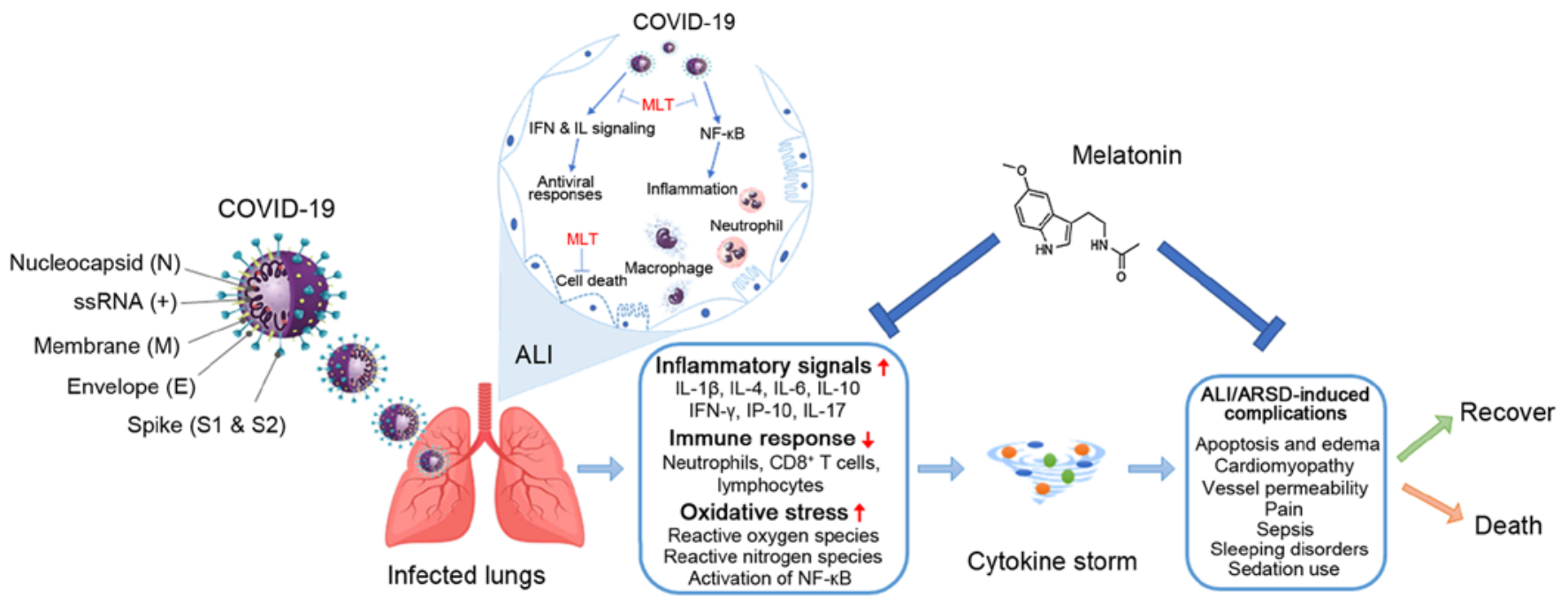

Figure 2. Possible mechanistic routes, in which melatonin could affect the infection process. Serious inflammation, oxidation, and an over-reactive immune system response contribute to COVID-19 pathology, leading to a cytokine storm causing ALI/ARDS, and frequently death, which could be reduced by the administration of melatonin. ALI, acute lung injury; ARDS, respiratory distress syndrome; ss, single strand; MLT, melatonin.

\section{Clinical trials using melatonin to treat COVID-19}

At present, there is a limited number of clinical trials, using melatonin as a bioactive substance to treat COVID-19; however, the results so far are promising (55). Randomized controlled clinical trials are the gold standard for clinical research and they are required to establish whether there is an actual clinical benefit in using melatonin to treat COVID-19. The following studies are indicative of the action of melatonin on the serious symptoms, which develop as a result of contracting COVID-19.

The augmenting rate of COVID-19 infection and associated death is of ultimate concern; therefore, it is important to eliminate the spread and that new clinical trials are designed to, not only ameliorate the pathological symptoms of COVID-19, but also to eliminate the high death rate (55). At present, there is not a promising and effective therapy, that is able to treat COVID-19.

Pharmaceutical research is focused on the investigation of novel drugs; however, this is time consuming, due to drug resistance and repeatedly occurring virus mutations (56-58). Furthermore, antiviral drugs are effective in mild to moderate cases of the virus, if used in early stages; however, they are not able to treat patients who have severe symptoms, due to overreaction of the innate immune response, which leads to destructive inflammation $(59,60)$. To overcome the complexity of viral infection, a symptomatic and non-virus-specific therapy should be considered (61).

Clinical trials have shown satisfactory safety results when melatonin was administered orally, at 3,6 and $10 \mathrm{mg}$ in patients in intensive care units (ICUs) in hospital (62-64). Furthermore, another clinical study has shown that there were no adverse reports when melatonin was used at a high dose of $1 \mathrm{~g} /$ day, for a month in humans (65). It has a high safety profile in humans; however, it is vital that melatonin should be controlled thoroughly when it is administered to patients with COVID-19 $(4,66)$.

In view of the fact that melatonin has been found to have effective anti-inflammatory and anti-oxidative properties, which are known to enhance the innate immune system and promote adaptive immunity $(51,55)$, it can be used as an adjuvant therapy in treating patients with COVID-19, with lower and predictive side effects compared to a novel drug, providing a supportive profile for a direct and safe clinical use in patients $(4,67,68)$. Furthermore, its safe use has been corroborated by the fact

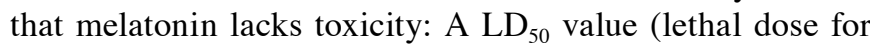
$50 \%$ of the animals used) could not be established. In addition, a high-dose of $800 \mathrm{mg} / \mathrm{kg}$ for bodyweight was not lethal (69). Studies, which used human subjects, and were treated with varying doses of melatonin (1-6.6 g/day) for 30-45 days, have concluded that, aside from drowsiness, all other side effects (headache, stomach cramps, persistent sleepiness and somnolence) were not apparent at the end of the test period $(65,70)$.

At present, there are some ongoing clinical trials, that have investigated the therapeutic dynamic of melatonin in treating COVID-19 (Table I). It has been observed that melatonin was only used in 11 clinical trials. The time spent on clinical trials, using a bioactive compound, such as melatonin, is not supported, due to the lack of protective patents for a natural compound (71). However, this obstacle has to be surpassed, not only due to its highly safe and effective profile, but also due to its multifactorial and synergic pharmacological actions $(15,72)$.

A clinical trial (EudraCT, 2020-001808-42) has been approved by the Spanish Agency of Medicines and Medical Devices (AEMPS), which allows the identification of the doses of melatonin, that can be effective in treating COVID-19 (73,74). This is a phase II, single center, double-blind, placebo-controlled randomized trial with a two-arm parallel group design and a 2:1 allocation ratio. The chosen participants are solely critically ill adult patients, that fulfill all of the inclusion criteria and none of the exclusion criteria. The anticipated main outcome is the mortality rate, in each study group, which is represented in frequency and time-to-event at day 28, after randomization.

Furthermore, the AEMPS has also approved another randomized clinical trial (EudraCT, 2020-001530-35) (75). This is a randomized multicenter clinical trial, which will 


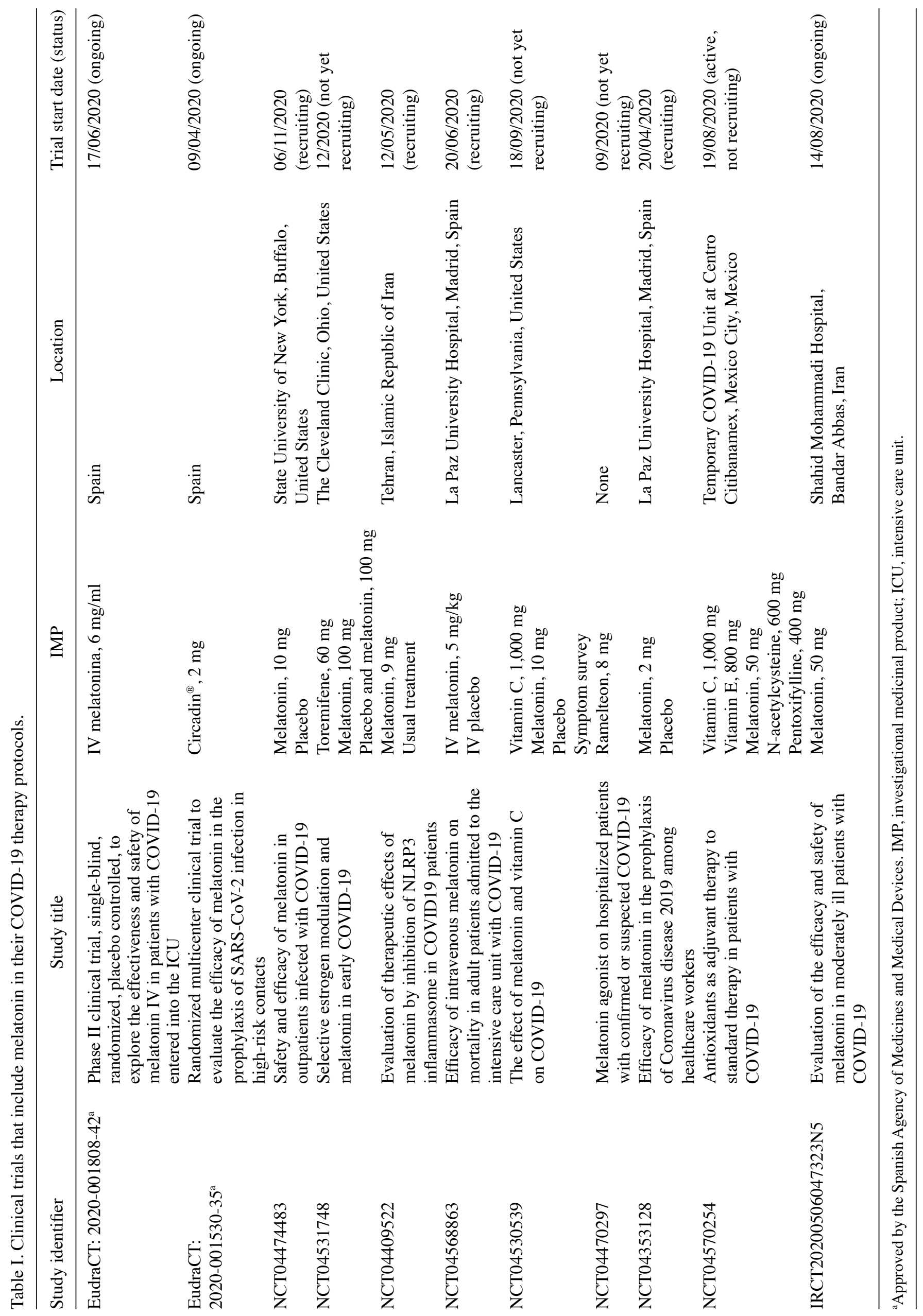


evaluate the efficacy of melatonin for the prophylaxis of SARS-CoV-2 infection, in patients who are high-risk, using 2 mg Circadin ${ }^{\circledR}$, as medication.

Another study (NCT04474483) is being undertaken at the State University of New York in Buffalo (USA), which is a pilot randomized, double-blind, placebo-controlled clinical trial (76). This clinical trial will evaluate the effect and safety of melatonin in out-patient adults, possibly infected with COVID-19. The purpose of this study is to assess the clinical profile of melatonin, compared with that in patients being treated with a placebo, with respect to COVID-19-related symptoms. It will also analyze the mortality rate and define whether the antioxidant and anti-inflammatory actions of melatonin are able to reduce the threat and severity of COVID-19 in a mild state infection.

The clinical trial, NCT04531748, is a clinical trial evaluating a 14-day intervention, with either a combination treatment of toremifene and melatonin or melatonin alone, in adults with mild COVID-19 (77). This study is double-blind, the allocation is randomized and assesses whether there is amelioration of symptoms (fatigue, fever, cough and dyspnea) and clinical signs, compared with that in patients being treated with the placebo, for 30 days.

Another clinical trial (NCT04409522), being undertaken in Iran, will use melatonin, as an anti-inflammatory treatment, when severe inflammation occurs in patients with COVID-19 (78). In this trial, the pharmacological role of melatonin, in inhibiting the inflammasome, NLRP3, a mechanism responsible for numerous serious inflammatory disorders, is examined. It has been hypothesized that measurements of melatonin levels in the human body are essential to characterize melatonin as a potential adjuvant in antiviral medical therapies to treat COVID-19.

In a melatonin-COVID-19 study (NCT04568863), being undertaken at the La Paz University Hospital (Madrid, Spain), which is a phase II, double-blind, single-center and randomized placebo-controlled clinical trial, the safety and efficacy of intravenously administered melatonin in patients with COVID-19, in the ICUs, is being investigated (79). The clinical condition of the patients with COVID-19 in the ICUs is characterized by a high risk of sepsis, respiratory malfunctions and death. This clinical trial intends on decreasing the mortality rate and ameliorating the patient's outcome by preventing the creation of free radicals and the development of sepsis.

There is another double-blind placebo-controlled clinical trial (NCT04530539), which has been developed at the Lancaster General Hospital (Pennsylvania, USA) and assesses the benefits of administering melatonin and vitamin $\mathrm{C}$ to patients with COVID-19 (80).

In another clinical trial for COVID-19 (NCT04470297) melatonin is being investigated as an antiapoptotic, antioxidative and anti-inflammatory therapy in treating acute lung injury (81). In this study, the medical effects of ramelteon $(8 \mathrm{mg})$, a melatonin receptor selective agonist, are being determined, and aims to minimize the risk of clinical downturn and the prolonged hospitalization of patients with COVID-19.

Melatonin is also being used in another clinical trial (NCT04353128), undertaken at the La Paz University Hospital (Spain), and intends to prevent the infection of COVID-19 in healthcare workers, who are at high risk (82). This is a randomized controlled trial, in which melatonin is being used for its anti-inflammatory and anti-oxidative properties, providing an effective shield to protect healthcare personnel. This investigation suggested that melatonin offers protection from severe COVID-19 symptoms, when administered daily, at $2 \mathrm{mg}$.

In addition, the Cleveland Clinic (Ohio, United States) published an observational study that analyzed 34 different drugs used to treat a variety of diseases, such as respiratory, cardiovascular, neurological, immunological and cancer, using computational tests (83). Out of these drugs, melatonin usage was found to lower the chance of a positive COVID-19 test by $28 \%$, which was confirmed using reverse transcription-quantitative PCR. The study also found that melatonin usage was associated with a $52 \%$ reduced likelihood of a positive COVID-19 result in African Americans. Furthermore, melatonin usage was associated with a reduced likelihood of a SARS-CoV-2 positive test result compared with that in the use of angiotensin II receptor blockers or angiotensin-converting enzyme inhibitors (83). However, as this was an observational study, its findings only suggest a possible association between melatonin usage and COVID-19 and not causation.

Furthermore, the ANTIOX-COVID (NCT04570254) study is a trial, which administers $5 \mathrm{mg}$ melatonin tablets, every $24 \mathrm{~h}$ (total amount $50 \mathrm{mg}$ ) to patients with or without septic shock, in a simultaneous administration of vitamin C, vitamin $\mathrm{E}, \mathrm{N}$-acetylcysteine and pentoxifylline (a known hemorheologic agent, which aids blood flow through narrowed arteries) (84). COVID-19 infection due to NO formation and reactive nitrogen species, may lead to severe pneumonia with pulmonary alveolar collapse. This trial is a non-randomized controlled study and intends to examine the results of a synergistic anti-oxidative effect of melatonin and pentoxifylline, aimed at regulating the uncontrolled and high oxidative stress provoked by COVID-19. In addition, this adjunct treatment, with pentoxifylline and antioxidants, lowers lipoperoxidation and oxidative stress, eliminating the requirement for ventilation in patients with or without septic shock and minimizes the SARS-COV2 pneumonia risk.

Lastly, the efficacy and safety of melatonin in patients who are moderately ill with COVID-19 was evaluated in Iran (Shahid Mohammadi Hospital; IRCT20200506047323N5) (85). This study primarily investigated the recovery rate in patients with clinical symptoms and the oxygen saturation, as well as, the improvement in the levels of serum inflammatory parameters (CRP, TNF- $\alpha$, IL-1 $\beta$, and IL-6). It also investigated the improvement of clinical and paraclinical symptoms, along with the incidence of serious adverse drug reactions (Table I) $(86,87)$.

\section{Conclusion}

The so called 'hormone of darkness', melatonin, due to its synthesis at night-time, has recently attracted the interest of infectious diseases specialists and epidemiologists, due to its adjuvant role in treating patients with COVID-19. The recent clinical trials, which involve melatonin, have been highlighted, collectively, in the present review, to the best of our knowledge, for the first time. However, more clinical studies are required, to understand the mechanism of action, at the molecular level, so that its multifactorial and synergic pharmacological actions, coupled with its high safety and effective profile, could be used to treat patients with COVID-19, and reduce the impact of the pandemic. 


\section{Acknowledgements}

SKK is indebted to the Onassis Foundation for supporting this work via the scholarship G ZO 011-1/ 2018-2019.

\section{Funding}

No funding was received.

\section{Availability of data and materials}

Not applicable.

\section{Authors' contributions}

MV contributed to the design of the study and supervised the writing of the manuscript. AS, AD and SKK retrieved the data and wrote the manuscript. IPP aided in data extraction and critically revised the manuscript. AS and AD designed Figs. 1 and 2. SKK and IPP edited and revised the manuscript. All authors have read and agreed to the final version of the manuscript. AS, AD, SKK and IPP confirm the authenticity of the data presented in this manuscript.

\section{Ethics approval and consent to participate}

Not applicable.

\section{Patient consent for publication}

Not applicable.

\section{Competing interests}

The authors declare that they have no competing interests.

\section{References}

1. Zhou Y, Wang F, Tang J, Nussinov R and Cheng F: Artificial intelligence in COVID-19 drug repurposing. Lancet Digit Heal 2: e667-e676, 2020.

2. Konstantinidou S and Papanastasiou I: Repurposing current therapeutic regimens against SARS-CoV-2 (Review). Exp Ther Med 2: 1845-1855, 2020.

3. Sidiropoulou P, Docea AO, Nikolaou V, Katsarou MS, Spandidos DA, Tsatsakis A, Calina D and Drakoulis N: Unraveling the roles of vitamin D status and melanin during COVID-19 (Review). Int J Mol Med 47: 92-100, 2020.

4. Zhang R, Wang X, Ni L, Di X, Ma B, Niu S, Liu C and Reiter RJ: COVID-19: Melatonin as a potential adjuvant treatment. Life Sci 250: 117583, 2020.

5. Turek FW and Gillette MU: Melatonin, sleep, and circadian rhythms: Rationale for development of specific melatonin agonists. Sleep Med 5: 523-532, 2004.

6. Zlotos DP: Recent advances in melatonin receptor ligands. Arch Pharm (Weinheim) 338: 229-247, 2005.

7. Jahanban-Esfahlan R, Mehrzadi S, Reiter RJ, Seidi K, Majidinia M, Baghi HB, Khatami N, Yousefi B and Sadeghpour A: Melatonin in regulation of inflammatory pathways in rheumatoid arthritis and osteoarthritis: Involvement of circadian clock genes. Br J Pharmacol 175: 3230-3238, 2018

8. Kratz EM and Piwowar A: Melatonin, advanced oxidation protein products and total antioxidant capacity as seminal parameters of prooxidant-antioxidant balance and their connection with expression of metalloproteinases in context of male fertility. J Physiol Pharmacol 68: 659-668, 2017.
9. Olcese JM: Melatonin and female reproduction: An expanding universe. Front Endocrinol (Lausanne) 11: 85, 2020.

10. Hosseinzadeh A, Javad-Moosavi SA, Reiter RJ, Yarahmadi R, Ghaznavi H and Mehrzadi S: Oxidative/nitrosative stress, autophagy and apoptosis as therapeutic targets of melatonin in idiopathic pulmonary fibrosis. Expert Opin Ther Targets 22: 1049-1061, 2018.

11. Hosseinzadeh A, Javad-Moosavi SA, Reiter RJ, Hemati K, Ghaznavi $\mathrm{H}$ and Mehrzadi S: Idiopathic pulmonary fibrosis (IPF) signaling pathways and protective roles of melatonin. Life Sci 201: 17-29, 2018.

12. Luchetti F, Canonico B, Betti M, Arcangeletti M, Pilolli F, Piroddi M, Canesi L, Papa S and Galli F: Melatonin signaling and cell protection function. FASEB J 24: 3603-3624, 2010.

13. Hill SM, Belancio VP, Dauchy RT, Xiang S, Brimer S, Mao L, Hauch A, Lundberg PW, Summers W, Yuan L, et al: Melatonin: An inhibitor of breast cancer. Endocr Relat Cancer 22: R183-R204, 2015.

14. Carlberg C: Gene regulation by melatonin. Ann N Y Acad Sci 917: 387-396, 2000.

15. Bahrampour Juybari K, Pourhanifeh MH, Hosseinzadeh A, Hemati K and Mehrzadi S: Melatonin potentials against viral infections including COVID-19: Current evidence and new findings. Virus Res 287: 198108, 2020.

16. Weaver SC, Ferro C, Barrera R, Boshell J and Navarro JC: Venezuelan equine encephalitis. Annu Rev Entomol 49: 141-174, 2004.

17. Schoneboom BA, Lee JS and Grieder FB: Early expression of IFN-alpha/beta and iNOS in the brains of Venezuelan equine encephalitis virus-infected mice. J Interferon Cytokine Res 20: 205-215, 2000

18. Schoneboom BA, Fultz MJ, Miller TH, McKinney LC and Glieder FB: Astrocytes as targets for Venezuelan equine encephalitis virus infection. J Neurovirol 5: 342-354, 1999.

19. Schoneboom BA, Catlin KMK, Marty AM and Grieder FB: Inflammation is a component of neurodegeneration in response to Venezuelan equine encephalitis virus infection in mice. J Neuroimmunol 109: 132-146, 2000.

20. Cain MD, Salimi H, Gong Y, Yang L, Hamilton SL, Heffernan JR, Hou J, Miller MJ and Klein RS: Virus entry and replication in the brain precedes blood-brain barrier disruption during intranasal alphavirus infection. J Neuroimmunol 308: 118-130, 2017.

21. Keck F, Kortchak S, Bakovic A, Roberts B, Agrawal N and Narayanan A: Direct and indirect pro-inflammatory cytokine response resulting from TC-83 infection of glial cells. Virulence 9: 1403-1421, 2018.

22. Valero N, Mosquera J, Alcocer S, Bonilla E, Salazar J and Álvarez-Mon M: Melatonin, minocycline and ascorbic acid reduce oxidative stress and viral titers and increase survival rate in experimental Venezuelan equine encephalitis. Brain Res 1622: 368-376, 2015.

23. Montiel M, Bonilla E, Valero N, Mosquera J, Espina LM, Quiroz Y and Álvarez-Mon M: Melatonin decreases brain apoptosis, oxidative stress, and CD200 expression and increased survival rate in mice infected by Venezuelan equine encephalitis virus. Antivir Chem Chemother 24: 99-108, 2015.

24. Valero N, MarinaEspina L, Bonilla E and Mosquera J: Melatonin decreases nitric oxide production and lipid peroxidation and increases interleukin-1 beta in the brain of mice infected by the Venezuelan equine encephalomyelitis virus. J Pineal Res 42: 107-112, 2007.

25. Valero N, Meleán E, Bonilla E, Arias J, Espina LM, Chacin-Bonilla L, Larreal Y, Maldonado M and Añez F: In vitro, melatonin treatment decreases nitric oxide levels in murine splenocytes cultured with the venezuelan equine encephalomyelitis virus. Neurochem Res 30: 1439-1442, 2005.

26. Tuñõn MJ, San-Miguel B, Crespo I, Laliena A, Vallejo D, Álvarez M, Prieto J and González-Gallego J: Melatonin treatment reduces endoplasmic reticulum stress and modulates the unfolded protein response in rabbits with lethal fulminant hepatitis of viral origin. J Pineal Res 55: 221-228, 2013.

27. Crespo I, Miguel BS, Laliena A, Álvarez M, Culebras JM, González-Gallego $\mathrm{J}$ and Tuñón MJ: Melatonin prevents the decreased activity of antioxidant enzymes and activates nuclear erythroid 2-related factor 2 signaling in an animal model of fulminant hepatic failure of viral origin. J Pineal Res 49: 193-200, 2010.

28. Verdonschot J, Hazebroek M, Merken J, Debing Y, Dennert R, Brunner-La RoccaHP and Heymans S: Relevance of cardiac parvovirus B19 in myocarditis and dilated cardiomyopathy: Review of the literature. Eur J Heart Fail 18: 1430-1441, 2016. 
29. Kühl U, Pauschinger M, Seeberg B, Lassner D, Noutsias M, Poller W and Schultheiss HP: Viral persistence in the myocardium is associated with progressive cardiac dysfunction. Circulation 112: 1965-1970, 2005.

30. Ouyang H, Zhong J, Lu J, Zhong Y, Hu Y and Tan Y: Inhibitory effect of melatonin on Mst1 ameliorates myocarditis through attenuating ER stress and mitochondrial dysfunction. J Mol Histol 50: 405-415, 2019.

31. Sang Y, Gu X, Pan L, Zhang C, Rong X, Wu T, Xia T, Li Y, Ge L, Zhang $\mathrm{Y}$ and $\mathrm{Chu} \mathrm{M}$ : Melatonin ameliorates coxsackievirus B3-induced myocarditis by regulating apoptosis and autophagy. Front Pharmacol 9: 1384, 2018.

32. Simko F, Hrenak J, Dominguez-Rodriguez A and Reiter RJ: Melatonin as a putative protection against myocardial injury in COVID-19 infection. Expert Rev Clin Pharmacol 13: 921-924, 2020

33. Junaid A, Tang H, van Reeuwijk A, Abouleila Y, Wuelfroth P, van Duinen V, Stam W, van Zonneveld AJ, Hankemeier T and Mashaghi A: Ebola hemorrhagic shock Syndrome-on-a-Chip. iScience 23: 100765, 2020

34. Martín Giménez VM, Inserra F, Tajer CD, Mariani J, Ferder L, Reiter RJ and Manucha W: Lungs as target of COVID-19 infection: Protective common molecular mechanisms of vitamin D and melatonin as a new potential synergistic treatment. Life Sci 254: 117808, 2020.

35. Hill-Batorski L, Halfmann P, Neumann G and Kawaoka Y: The cytoprotective enzyme heme Oxygenase-1 suppresses ebola virus replication. J Virol 87: 13795-13802, 2013.

36. Hazra S, Chaudhuri AG, Tiwary BK and Chakrabarti N: Matrix metallopeptidase 9 as a host protein target of chloroquine and melatonin for immunoregulation in COVID-19: A network-based meta-analysis. Life Sci 257: 118096, 2020.

37. Habtemariam S, Daglia M, Sureda A, Selamoglu Z, Fuat Gulhan M and Mohammad Nabavi S: Melatonin and respiratory diseases: A review. Curr Top Med Chem 17: 467-488, 2016.

38. Shim DW, Shin HJ, Han JW, Ji YE, Jang CH, Koppula S, Kang TB and Lee KH: A novel synthetic derivative of melatonin, 5-hydroxy-2'-isobutyl-streptochlorin (HIS), inhibits inflammatory responses via regulation of TRIF-dependent signaling and inflammasome activation. Toxicol Appl Pharmacol 284: 227-235, 2015.

39. Reiter RJ, Sharma R, Ma Q, Dominquez-Rodriguez A, Marik PE and Abreu-Gonzalez P: Melatonin inhibits COVID-19-induced cytokine storm by reversing aerobic glycolysis in immune cells: A mechanistic analysis. Med Drug Discov 6: 100044, 2020.

40. Bouhafs RKL and Jarstrand C: Effects of antioxidants on surfactant peroxidation by stimulated human polymorphonuclear leukocytes. Free Radic Res 36: 727-734, 2002.

41. Zhang Y, Li X, Grailer JJ, Wang N, Wang M, Yao J, Zhong R, Gao GF, Ward PA, Tan DX and Li X: Melatonin alleviates acute lung injury through inhibiting the NLRP3 inflammasome. J Pineal Res 60: 405-414, 2016.

42. Soussia I Ben, Mies F, Naeije R and Shlyonsky V: Melatonin down-regulates volume-sensitive chloride channels in fibroblasts. Pflugers Arch 464: 273-285, 2012.

43. Farhood B, Aliasgharzadeh A, Amini P, Rezaeyan A, Tavassoli A Motevaseli E, Shabeeb D, Musa AE and Najafi M: Mitigation of radiation-induced lung pneumonitis and fibrosis using metformin and melatonin: A histopathological study. Medicina (Kaunas) 55: $417,2019$.

44. Hong S, Kim CY, Lee JEand Seong GJ: Agmatine protects cultured retinal ganglion cells from tumor necrosis factor-alpha-induced apoptosis. Life Sci 84: 28-32, 2009.

45. Bosco AD, Schedler FB, Colares JR, Schemitt EG, Hartmann RM, Forgiarini Junior LA, Dias AS and Marroni NP: Melatonin effects on pulmonary tissue in the experimental model of hepatopulmonary syndrome. J Bras Pneumol 45: e20170164, 2019 (In English, Portuguese).

46. Pita R, Marco-Contelles J, Ramos E, Del Pino J and Romero A Toxicity induced by chemical warfare agents: Insights on the protective role of melatonin. Chem Biol Interact 206: 134-142, 2013.

47. Macit E, Yaren H, Aydin I, Kunak ZI, Yaman H, Onguru O, Uysal B, Korkmaz A, Turel S and Kenar L: The protective effect of melatonin and S-methylisothiourea treatments in nitrogen mustard induced lung toxicity in rats. Environ Toxicol Pharmacol 36: 1283-1290, 2013.

48. Llinàs L, Peinado VI, Ramon Goñi J, Rabinovich R, Pizarro S, Rodriguez-Roisin R, Barberà JA and Bastos R: Similar gene expression profiles in smokers and patients with moderate COPD. Pulm Pharmacol Ther 24: 32-41, 2011.
49. Al-Rasheed NM, Fadda L, Attia HA, Sharaf IA, Mohamed AM and Al-Rasheed NM: Pulmonary prophylactic impact of melatonin and/or quercetin: A novel therapy for inflammatory hypoxic stress in rats. Acta Pharm 67: 125-135, 2017.

50. Jin H, Wang Y, Zhou L, Liu L, Zhang P, Deng W and Yuan Y: Melatonin attenuates hypoxic pulmonary hypertension by inhibiting the inflammation and the proliferation of pulmonary arterial smooth muscle cells. J Pineal Res 57: 442-450, 2014.

51. Hardeland R: Aging, melatonin, and the pro-and anti-inflammatory networks. Int J Mol Sci 20: 1233, 2019.

52. Sehirli AO, Sayiner S and Serakinci N: Role of melatonin in the treatment of COVID-19; as an adjuvant through cluster differentiation 147 (CD147). Mol Biol Rep 47: 8229-8233, 2020.

53. Wu GC, Peng CK, Liao WI, Pao HP, Huang KL and Chu SJ: Melatonin receptor agonist protects against acute lung injury induced by ventilator through up-regulation of IL-10 production. Respir Res 21: 65, 2020.

54. Al-Zaqri N, Pooventhiran T, Alsalme A, Warad I, John AM and Thomas R: Structural and physico-chemical evaluation of melatonin and its solution-state excited properties, with emphasis on its binding with novel coronavirus proteins. J Mol Liq 318: $114082,2020$.

55. Kleszczyński K, Slominski AT, Steinbrink K and Reiter RJ: Clinical trials for use of melatonin to fight against COVID-19 are urgently needed. Nutrients 12: 2561, 2020.

56. Wang R, Hozumi Y, Yin C and Wei GW: Mutations on COVID-19 diagnostic targets. Genomics 112: 5204-5213, 2020.

57. Shariare MH, Parvez MAK, Karikas GA and Kazi M: The growing complexity of COVID-19 drug and vaccine candidates: Challenges and critical transitions. J Infect Public Health 14: 214-220, 2021.

58. Shneider A, Kudriavtsev A and Vakhrusheva A: Can melatonin reduce the severity of COVID-19 pandemic? Int Rev Immunol 39: 153-162, 2020

59. Shi J, Xiao Y, Zhang Y, Geng D, Cong D, Shi KX and Knapp RJ: Challenges of drug development during the COVID-19 pandemic: Key considerations for clinical trial designs. Br J Clin Pharmacol: Oct 29, 2020 (Epub ahead of print).

60. Parvathaneni V and Gupta V: Utilizing drug repurposing against COVID-19-Efficacy, limitations, and challenges. Life Sci 259: 118275,2020

61. Acuña-Castroviejo D, Escames G, Figueira JC, de la Oliva P, Borobia AM and Acuña-Fernández C: Clinical trial to test the efficacy of melatonin in COVID-19. J Pineal Res 69: e12683, 2020.

62. Bourne RS, Mills GH and Minelli C: Melatonin therapy to improve nocturnal sleep in critically ill patients: Encouraging results from a small randomised controlled trial. Crit Care 12: R52, 2008

63. Mistraletti G, Umbrello M, Sabbatini G, Miori S, Taverna M, Cerri B, Mantovani ES, Formenti P, Spanu P, D'Agostino A, et al: Melatonin reduces the need for sedation in ICU patients: A randomized controlled trial. Minerva Anestesiol 81: 1298-1310, 2015.

64. Mistraletti G, Sabbatini G, Taverna M, Figini MA, Umbrello M, Magni P, Ruscica M, Dozio E, Esposti R, DeMartini G, et al: Pharmacokinetics of orally administered melatonin in critically ill patients. J Pineal Res 48: 142-147, 2010.

65. Nordlund JJ and Lerner AB: The effects of oral melatonin on skin color and on the release of pituitary hormones. J Clin Endocrinol Metab 45: 768-774, 1977.

66. Biancatelli RMLC, Berrill M, Mohammed YH and Marik PE: Melatonin for the treatment of sepsis: The scientific rationale. J Thorac Dis 12 (Suppl 1): S54-S65, 2020.

67. Herrera EA and González-Candia A: Comment on melatonin as a potential adjuvant treatment for COVID-19. Life Sci 253: 117739,2020

68. Moghadam SO: A review on currently available potential therapeutic options for covid-19. Int J Gen Med 13: 443-467, 2020.

69. Barchas J, DaCosta F and Spector S: Acute Pharmacology of melatonin. Nature 214: 919-920, 1967.

70. Papavasiliou PS, Cotzias GC, Duby SE, Steck AJ, Bell M and Lawrence WH: Melatonin and Parkinsonism. JAMA 221: 88 , 1972.

71. Pandi-Perumal SR, Cardinali DP, Reiter RJ and Brown GM: Low melatonin as a contributor to SARS-CoV-2 disease. Melatonin Res 3: 558-576, 2020.

72. Öztürk G, Akbulut KG and Güney S: Melatonin, aging, and COVID-19: Could melatonin be beneficial for COVID-19 treatment in the elderly? Turkish J Med Sci 50: 1504-1512, 2020. 
73. EU Clinical Trials Register: Eudract number: 2020-001808-42. https://www.clinicaltrialsregister.eu/ctr-search/trial/ 2020-001808-42/ES. Accessed December 5, 2020.

74. Rodríguez-Rubio M, Figueira JC, Acuña-Castroviejo D, Borobia AM, Escames G and de La Oliva P: A phase II single-center, double-blind, randomized placebo-controlled trial to explore the efficacy and safety of intravenous melatonin in patients with COVID-19 admitted to the intensive care unit (MelCOVID study): A structured summary of a study protocol for a randomized controlled trial. Trials 21 : 699, 2020.

75. EU Clinical Trials Register: Eudract_number: 2020-001530-35 https://www.clinicaltrialsregister.eu/ctr-search/trial/ 2020-001530-35/ES. Accessed December 5, 2020.

76. ClinicalTrials.gov: Safety and Efficacy of Melatonin in Outpatients Infected With COVID-19. https://clinicaltrials. gov/ct2/show/NCT04474483?term=melatonin\&cond=Covid19\& $\mathrm{draw}=2 \& \mathrm{rank}=1$. Accessed December 5, 2020.

77. ClinicalTrials.gov: Selective Estrogen Modulation and Melatonin in Early COVID-19. ttps://clinicaltrials.gov/ct2/show/NCT04531748?t erm $=$ melatonin $\&$ cond $=$ Covid19\&draw $=2 \&$ rank $=2$. December 5 , 2020.

78. ClinicalTrials gov: Evaluation of Therapeutic Effects of Melatonin by Inhibition of NLRP3 Inflammasome in COVID19 Patients. https://clinicaltrials.gov/ct2/show/NCT04409522?te $\mathrm{rm}=$ melatonin $\&$ cond $=$ Covid $19 \& \mathrm{draw}=1 \& \mathrm{rank}=3$. Accessed December 5, 2020.

79. ClinicalTrials.gov: Efficacy of Intravenous Melatonin on Mortality in Adult Patients Admitted to the Intensive Care Unit With COVID-19. https://clinicaltrials.gov/ct2/show/NCT0456886 3 ?term $=$ melatonin $\&$ cond $=$ Covid $19 \& d r a w=1 \&$ rank $=4$. Accessed December 5, 2020.

80. ClinicalTrials.gov: The Effect of Melatonin and Vitamin C on COVID-19. https://clinicaltrials.gov/ct2/show/NCT04530539? term $=$ melatonin $\&$ cond $=$ Covid $19 \&$ draw $=1 \&$ rank $=5$. Accessed December 5, 2020.
81. ClinicalTrials.gov: Melatonin Agonist on Hospitalized Patients With Confirmed or Suspected COVID-19. https://clinicaltrials. gov/ct $2 /$ show $/$ NCT04470297?term $=$ melatonin\&cond=Covid19\& draw $=1 \&$ rank=6. Accessed December 5, 2020.

82. ClinicalTrials.gov: Efficacy of Melatonin in the Prophylaxis of Coronavirus Disease 2019 (COVID-19) Among Healthcare Workers. https://clinicaltrials.gov/ct2/show/NCT04353128?te $\mathrm{rm}=$ melatonin \& cond $=$ Covid $19 \& \mathrm{draw}=1 \& \mathrm{rank}=7$. Accessed December 5, 2020.

83. Zhou Y, Hou Y, Shen J, Mehra R, Kallianpur A, Culver DA, Gack MU, Farha S, Zein J, Comhair S, et al: A network medicine approach to investigation and population-based validation of disease manifestations and drug repurposing for COVID-19. PLoS Biol 18: e3000970, 2020.

84. ClinicalTrials.gov: Antioxidants as Adjuvant Therapy to Standard Therapy in Patients With COVID-19. https://clinicaltrials.gov/ct $2 /$ show $/$ NCT0 4570254 ?term $=$ melatonin \& cond $=$ Covid19\&draw $=1 \&$ rank=8. Accessed December 5, 2020.

85. Hassaniazad M, Bazram A, Hassanipour S and Fathalipour M: Evaluation of the efficacy and safety of favipiravir and interferon compared to lopinavir/ritonavir and interferon in moderately ill patients with COVID-19: A structured summary of a study protocol for a randomized controlled trial. Trials 21: 886, 2020.

86. ClinicalTrials.gov: Search of: Melatonin|Covid19-List Results. https://clinicaltrials.gov/ct2/results?recrs=\&cond=Covid19\&term = melatonin $\&$ cntry $=\&$ state $=\&$ cit $y=\&$ dist $=$. Accessed December 5 , 2020.

87. EU Clinical Trials Register: Search for covid-19 and melatonin. https://www.clinicaltrialsregister.eu/ctr-search/search?query=co vid-19+and+melatonin. Accessed December 5, 2020.

This work is licensed under a Creative Commons Attribution-NonCommercial-NoDerivatives 4.0 International (CC BY-NC-ND 4.0) License. 